\title{
Überlebensstrategien oder Lebensformen? Soziale und wirtschaftliche Verflechtungen im Alltag von Slumbewohnern in Klong Toey, Bangkok ${ }^{1}$
}

\section{Einleitung}

Leitmotiv der meisten Entwicklungsbemühungen ist die Überwindung von Unterentwicklung und Armut. So unterschiedlich die dazu entwickelten Theorien in Ideologie und Methoden auch sind, die meisten weisen zumindest eine Parallele auf: Sie argumentieren mit reduktionistischen Verallgemeinerungen (vgl. MENZEL, 1992). Unter solche Generalisierungen fallen zunächst die zu breit gefaßten Untersuchungseinheiten. Einerseits vermögen Meßgrößen wie "Bruttosozialprodukt pro Kopf» oder "Durchschnittliche Wachstumsraten», oft verwendet als Indikatoren für sozioökonomische Prosperität einer $\mathrm{Ge}$ sellschaft, Verteilungskriterien innerhalb derselben nicht zu berücksichtigen. Zudem werden nicht selten auch Kategorien wie "Wirtschaftssektoren», «Dorfgemeinschaften» oder "Berufsgruppen» als relevante Einheiten festgelegt, womit, durch Suggerieren von deren Homogenität, mögliche interne Ungleichgewichte verwischt werden.

Aber auch die Wahl von noch so kleinen Untersuchungsgrößen garantiert noch nicht, der gelebten Realität von Armut und Unterentwicklung näherzukommen, wenn die Analyse auf stark generalisierenden Modellen menschlicher Handlungsweisen (wie beispielsweise dem Modell des Homo oeconomicus) basiert. Solche Modelle bauen meist auf stark verallgemeinernden Grundprämissen als fix angenommenen Rahmenbedingungen auf, deren Abweichungen zur gelebten Realität in vielen Forschungsarbeiten nicht in Frage gestellt werden.

Aus Forschungsergebnissen, die auf solcherlei Generalisierungen aufbauen, werden nicht selten normative Maßnahmenkataloge direkt abgeleitet. Dabei wird jedoch oft übersehen, daß bereits der Festlegung von Meßgrößen, Kategorien oder Modellen vereinfachende Annahmen zugrunde liegen. Werden diese nun in konkrete Entwicklungspolitik umgesetzt, werden künstlich homogenisierte Kategorien und vereinheitlichte Rahmenbedingungen als real betrachtet, die in Wirklichkeit viel komplexerer Natur sind. Nicht zuletzt deshalb sind viele der gelenkten Entwicklungsinterventionen zum Scheitern verurteilt, da auf diese Weise wichtige Informationen verlorengehen und wesentliche Zusammenhänge vernachlässigt werden.

Bielefelder Entwicklungssoziologen ${ }^{2}$ haben die Problematik solcher zu stark verallgemeinernden Makroperspektiven erkannt und sind seit Ende der siebziger Jahre darum bemüht, das Verständnis von Armut und Unter- entwicklung mittels Ergänzungen auf der Mikroebene zu verbessern. Sie versuchen, die durch die Distanz der Makrotheorien verlorengehenden wirtschaftlichen und sozialen Faktoren aus der Realität des täglichen Lebens mit in die Betrachtung einzubeziehen, indem sie die das Überleben sichernden Tätigkeiten bedürftiger Individuen als Ausgangspunkt ihrer Forschungsarbeit wählen (EVERS. 1987: 137).

Von zentraler Bedeutung sind in ihren Analysen die Kombinationen und engen Verflechtungen verschiedenartiger Produktionsformen einer "Produktionseinheit» (beispielsweise eines Individuums, Haushalts oder Betriebs). Die Produktionsformen werden unterschieden in «Subsistenzproduktion» (Produktion für den Eigenbedarf) und "marktorientierte Produktion». Zur ersteren gehören beispielsweise unbezahlte Haushaltsarbeiten, die Erstellung von Wohnraum durch Eigenleistung oder der Anbau von Nahrungsmitteln für den Eigenkonsum. Die marktorientierte Produktion wird weiter unterteilt in formelle (offiziell registrierte) und informelle (nicht registrierte) produktive Tätigkeiten. ${ }^{3}$ Letztere werden vom Staat weder geschützt, noch sind sie diesem gegenüber verpflichtet. Als Beispiele gelten nicht erfaßter Handel von selbst hergestellten Gütern oder Lohnarbeit ohne Arbeitsvertrag.

Mit Hilfe dieser Kategorien läßt sich eine der Kernaussagen des Bielefelder Ansatzes formulieren: Bedürftige Bevölkerungsgruppen vermögen ihr Überleben meist nur durch eine Kombination von verschiedenartigen Produktionsformen zu sichern. Einkommens- oder Unterhaltsausfälle in einem Bereich sollen durch entsprechende Kompensationen in einem anderen abgefedert werden. (EVERS, 1987: 136 f.). Bedürftige Individuen oder Haushalte sind deshalb stets darum bemüht, ihr Überleben durch konstantes strategisches Handeln zu sichern. Die Erhaltung menschlichen Lebens hängt also von den durch die Produktionseinheiten gewählten Überlebensstrategien $\mathrm{ab}$, welche sich in verschiedensten Kombinationen von miteinander verflochtenen Produktionsformen äußern. (ELWERT, G./EVERS, H.-D./WILKENS, W. 1983: 284).

Marco Pronk, dipl. Geograph, Geographisches Institut der Universität Zürich Irchel, Winterthurerstraße 190 8057 Zürich 
Auf den folgenden Seiten werden Erkenntnisse über Verflechtungen im Alltag von Bewohnern des Slum Klong Toey in Bangkok diskutiert. Zwei wichtige Befunde seien an dieser Stelle vorweggenommen: Die empirischen Untersuchungen zeigten, daß erstens die Analyse der Produktionsformen allein nicht ausreicht, um Formen der Existenzsicherung angemessen zu erklären - auch «nicht-produktive» Tätigkeiten und soziale Beziehungen sind von zentraler Bedeutung. Zweitens ist die Bedeutung, welche die Handelnden ihren Tätigkeiten selbst beimessen, zu berücksichtigen, um nicht Gefahr zu laufen, jede einzelne Aktivität als Element einer «Überlebensstrategie» zu taxieren. Deshalb muß das soziale Umfeld des Slums als der die Handlungen und Motive mitstrukturierende Rahmen in die Betrachtung mit einbezogen werden. Ein kurzer Einblick in die Geschichte des Slums und seiner Bewohner soll helfen, die heutigen Verhältnisse zu verstehen.

\section{Zur Entstehungsgeschichte des Slums Klong Toey}

Mit rund einhunderttausend Einwohnern gilt der Slum Klong Toey heute als der größte Slumkomplex von Bangkok. Situiert auf einem ungenutzten Grundstück der staatlichen Hafenbehörde, ist seine Entstehungs- und Entwicklungsgeschichte eng mit jener des angrenzenden Hafenbetriebs verknüpft. Als zu Beginn der fünfziger Jahre die Fertigstellung des Hafenareals forciert wurde und damit eine größere Anzahl von Bauarbeitern angeheuert wurde, erlaubte die «Port Authority of Thailand» (PAT) als Grundbesitzerin das Errichten von provisorischen Temporärunterkünften auf ihrem Grundstück. Nach Beendigung der Konstruktionsarbeiten im Jahre 1954 und der Inbetriebnahme des seither wichtigsten Hafens Thailands wurden viele der ehemaligen Bauarbeiter als Kulis (Lastträger) für Trägerarbeiten angeworben. Viele von ihnen berichteten in ihren Heimatdörfern von den dauerhaften Verdienstmöglichkeiten im Hafen, worauf ihnen nach und nach Familienangehörige und Bekannte nach Klong Toey folgten. Vorläufig waren die Squatter (illegale Siedler) auf dem mit Tümpeln durchzogenen, sumpfigen Gelände geduldet.

Mit aufkommenden Handelsaktivitäten in Hafennähe wurde eine kommerzielle Nutzung des Bodens für die Hafenbehörde allmählich lukrativ. In der Folge wurden zahlreiche Gruppen von Siedlern von den Grundstücken vertrieben, die entweder an Geschäftsleute verpachtet oder für Bauprojekte beansprucht wurden. In der Regel ließen sich die Bewohner, denen kein Ersatz für ihre niedergewalzten Behausungen angeboten wurde, wieder auf einem noch freien Fleck des Hafengrundstücks nieder. Einzelne Gruppen begannen allmählich einen Widerstand gegen weitere Räumungen zu organisieren. Mit der Unterstützung von Angehörigen der Soziologischen Fakultät der Thammasat-Universität Bangkok gelang es, den dank der nun rege betriebenen Öffentlichkeitsarbeit entstandenen Druck auf die Hafenbehörden zu nutzen: 1973 wurde erstmals ein Vertreibungsvorhaben verhin- dert. In der Folge wurde ein Kompromiß ausgearbeitet, jeder umzusiedelnden Familie ein neues Stück Land zur Verfügung zu stellen. Von nun an konnte die PAT keine ersatzlosen Räumungen mehr durchführen, da der Widerstand der Squatter nun gut organisiert war und in der Öffentlichkeit breite Unterstützung fand. Die PAT suchte nach Verbündeten und fand vorerst in der «National Housing Authority" (NHA) eine Koalitionspartnerin. Die NHA war gerade neu gegründet worden, um mit der Errichtung von Billigwohnblöcken der desolaten Wohnsituation in Bangkok zu begegnen. Ihrer damaligen Politik entsprechend, verfolgte die NHA in Klong Toey das Ziel, mittelfristig die gesamte Slumbevölkerung in Mietskasernen umzuquartieren. Mit dem Einverständnis der PAT wurden in der Zeit von 1974 bis 1978 achtzehn solcher Wohnblöcke errichtet. Hier sollten in erster Linie jene Bewohner untergebracht werden, die dem Bau einer Expreßstraße und der Mietskasernen selbst weichen mußten.

Im Zeichen der massiv zunehmenden Außenhandelsaktivitäten plante die thailändische Regierung Anfang der achtziger Jahre ein Großprojekt im Hafenareal, unter anderem einen viel Platz beanspruchenden Containerterminal. Der gesamte Slumkomplex wäre nach diesem Vorhaben aufgelöst worden, hätten sich Slumbewohner mit Unterstützung der NHA und der Weltbank nicht vehement gegen dieses Vorhaben gewehrt. Nach zähen Verhandlungen konnten sich die Parteien auf ein redimensioniertes Konzept einigen, welches sich auf die Umsiedlung lediglich eines Teils des Slums beschränkte. Neu war, daß den in die nach ihrer Fläche benannte Siedlung "70 Rai» ${ }^{4}$ umzusiedelnden Familien ein vertraglich gesichertes Wohnrecht für zwanzig Jahre garantiert wurde. Als sich 1989 der amtierende Premierminister Chatichai Choonhavan nach einem Augenschein im Slum Klong Toey für eine verbesserte Wohnsituation einsetzen wollte, stieß er auf großen Widerstand sowohl seitens der Slumbewohner als auch der PAT. Sein Vorschlag umfaßte einerseits Wohnungsneubauten auf dem Slumareal, gegen welche die PAT ihr Veto wegen Zweckentfremdung des Grundstücks einlegte. Andererseits sollte einem Teil der Siedler ein Stück Land in der Provinz Prachinburi, östlich von Bangkok, zur Verfügung gestellt werden, was unter der Slumbevölkerung Protest hervorrief, weil diese ihre Verdienstmöglichkeiten im Hafen gefährdet sah. Der Plan wurde daraufhin widerrufen.

Auch spätere Umsiedlungsvorhaben mit dem Ziel, Slumbewohnern Grundstücke in anderen Gegenden inneroder außerhalb Bangkoks zur Verfügung zu stellen, sind am Widerstand der Squatter gescheitert und konnten bis heute nicht realisiert werden (PORT AUTHORITY: 3-9, SAMAPHAN CHUMCHON-AE-AD KLONG TOEY 1992: 8-11). Die Geschehnisse der vergangenen vier Dekaden im und um den Slum Klong Toey strukturierten diesen zu einem komplexen Gebilde. Die Tätigkeiten seiner Bewohner waren und sind eingebettet in Prozesse, Entscheidungen und Aktivitäten unterschiedlicher Tragweite: Einerseits hatten sich die Siedler an den Konsequenzen internatio- 
naler Handelsaktivitäten, staatlicher Entwicklungspolitik und städtischer Wohnbaumaßnahmen zu orientieren. Nicht zuletzt deshalb haben die Bewohner von Klong Toey im Laufe der Zeit Organisationsformen entwickelt, die ihnen gegen außen ein kollektives Auftreten im Widerstand gegen Umsiedlungsprojekte ermöglichten. Andererseits bildeten sich, den in den verschiedenen Quartieren vorherrschenden unterschiedlichen Rahmenbedingungen entsprechend, differenzierte Formen von Lebensweisen und nachbarschaftlichen Beziehungen. Diese sind von großer Bedeutung für existenzsichernde Tätigkeiten der Slumbewohner.

\section{Der Slum Klong Toey heute}

Die in den Umsiedlungsprogrammen seitens der PAT und NHA erklärte Absicht, den Slumbewohnern verbesserte Lebensbedingungen in Form von Wohnblöcken zu bieten, ist weitgehend fehlgeschlagen. Gemäß Schätzungen von Vertretern der Slum Klong Toey Federation lebten im Jahre 1992 dort nur noch 20 bis 30 Prozent der ursprünglich umgesiedelten Slumbewohner. Viele zogen erst gar nicht ein, andere sind nach kurzer Zeit wieder ausgezogen, da sich das Mietrecht auf solche Wohnungen zu attraktiven Preisen übertragen läßt: Im Untersuchungsjahr wurden die Wohnungen für bis zu 300000 Baht (ca. 15000 Fr.) ${ }^{5}$ gehandelt. ${ }^{6}$ Während in die Wohnungen in erster Linie Neuzuzüger mittlerer Einkommensschichten Einzug halten, investieren die Slumbewohner ihr leichtverdientes Geld vorwiegend in Konsumgüter und Baumaterialien, mit denen sie auf freiem Land eine neue Behausung aufbauen.

Ähnliche Schlüsse lassen sich aus dem erwähnten Umsiedlungsprojekt "70 Rai» ziehen: In den meist zweistöckigen, gemauerten Häusern wohnen nur noch rund 40 Prozent der Familien, denen ursprünglich das Einzugsrecht zugesprochen wurde. 1992 wurde hier bis zu einer Million Baht (ca. 50000 Fr.) für das Mietrecht bezahlt?

Die hohen Geldsummen, welche Slumbewohner durch Mietrechtsübertragungen erhandeln können, vermögen das Versagen der Umsiedlungspolitik allerdings nicht befriedigend zu erklären. Denn schon in den Planungsphasen entwickeln die Siedler großen Widerstand gegen solche Vorhaben und versuchen mit allen Mitteln, ihre momentane Wohnsituation zu erhalten. Oft sind es vor allem die gewohnten Lebensformen und überlebenssichernden Produktionstätigkeiten, welche die Slumbewohner nicht preisgeben wollen.

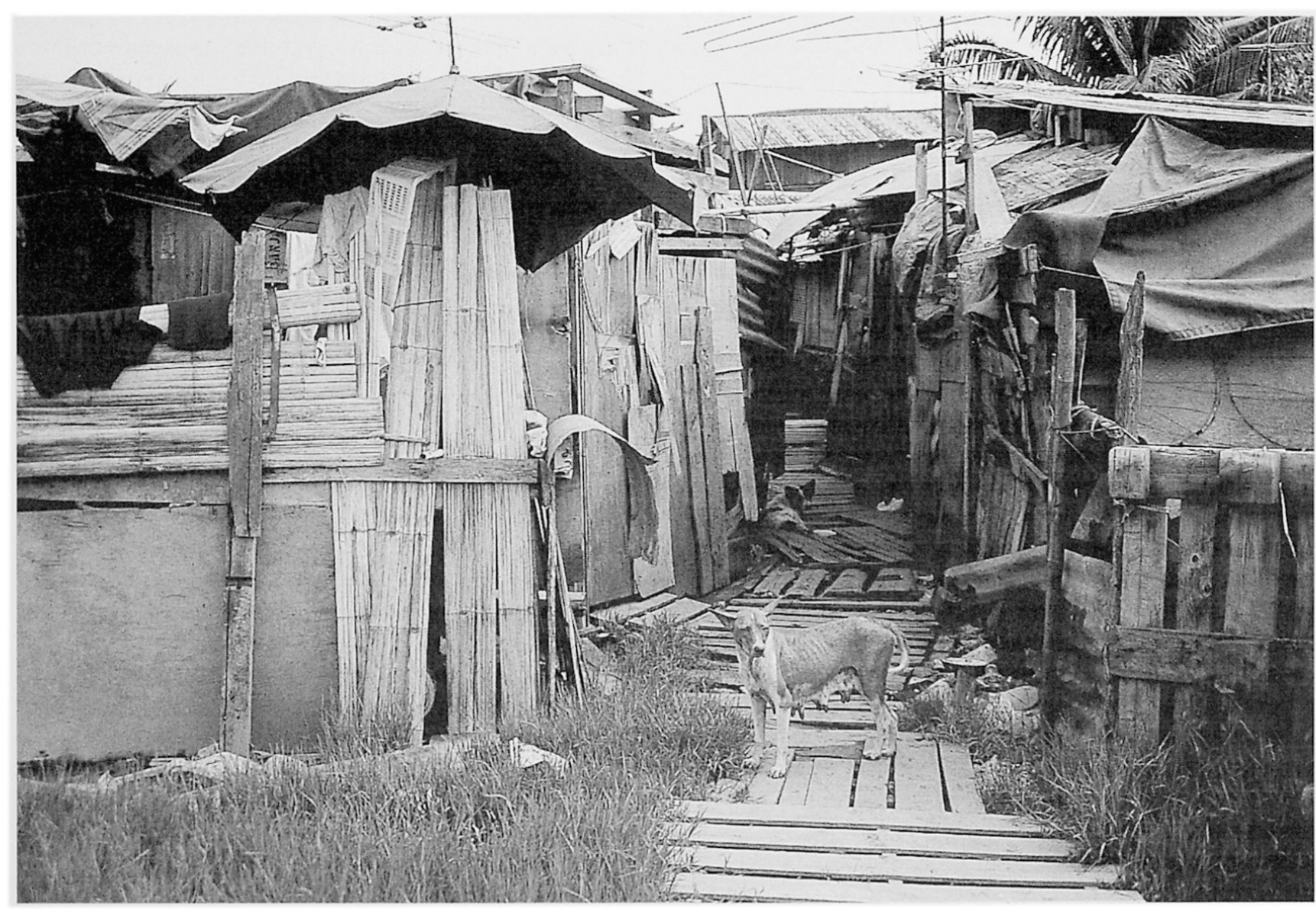

Abb.1 Die flexible Bauweise der Hütten ermöglicht bei Bedarf Aus- oder Umbauten. 
Der größte Teil der Bewohner von Klong Toey lebt in einfachen, meist unter eigener Regie behelfsmäßig aus Holz, Hartkarton und Blech hergestellten Hütten. Auf dem sumpfigen Untergrund stehen diese meist auf Pfählen, die Schutz vor Hochwasser bieten und für angemessene Durchlüftung der Behausungen sorgen. Diese sind oft so dicht nebeneinander gebaut, daß mit Leichtigkeit mal eine Pfanne, eine Handvoll Zwiebeln oder ein Schraubenzieher durch Fenster oder Ritzen in die Nachbarswohnung gereicht werden können. Die dünnen Wände ermöglichen die Unterhaltung mit Nachbarn während der Erledigung von Hausarbeiten. Die Kontakte zu Nachbarn sind dementsprechend intensiv. Ein hoher Informationsfluß und ein Gefühl der Kontrolle über das Geschehen in der näheren Umgebung sind die Folge. Die engen Beziehungen haben einen hohen Stellenwert im Alltag: Bei kleinen alltäglichen Diensten, seien es Reparaturarbeiten am Haus, Babysitten oder Ausleihen von Haushaltgeräten, ist die unentgeltliche Nachbarschaftshilfe selbstverständlich. ${ }^{8}$ Andererseits mischen sich Nachbarn auch in Erziehungsfragen ein, rügen Eltern für die Vernachlässigung der Kinder oder verweigern bei tadelhaftem Benehmen gar ihre nachbarschaftliche Kooperation. Die Einfachheit der Hütten birgt zudem aus der Sicht ihrer Bewohner noch weitere Vorteile in sich: Bei Bedarf, beispielsweise im Falle von Besuch oder Familienzuwachs, können diese mit einfachen Mitteln weiter unterteilt oder ausgebaut werden.

Nicht bei allen in den Hüttensiedlungen lebenden Bevölkerungsgruppen ist diese Lebensweise gleich beliebt. Insbesondere Angehörige der jüngeren Generation scheinen sich zunehmend diesem Druck sozialer Kontrolle entziehen zu wollen und stehen einem Umzug in die Wohnblöcke prinzipiell nicht abgeneigt gegenüber. Sie sehen dort in erster Linie die Chance, freier und unbekümmerter leben zu können. Nach Zwangsumsiedlungen kommen allerdings die wenigsten in den Genuß eines solchen Freiheitsgefühls: Einem Haushalt wird nämlich genau eine Wohnung zugewiesen, unabhängig von der Haushaltsgröße. Für größere Familien sind die Einheitswohnungen zu klein, und die Betonwände lassen keinen Aus- oder Umbau mehr zu. Außerdem werden nicht selten enge Freundschaftsbeziehungen unfreiwillig auseinandergerissen. Der größere Teil der Slumbevölkerung zieht demnach die Sicherheit ihrer angestammten Nachbarschaft den Wohnsilos vor: Sie fürchten sich vor der Unüberblickbarkeit der Treppenhäuser und Fluren der Apartmentbauten und den abgeschlossenen Wohnungen, die nachbarschaftliche Beziehungen erschweren.

Hinzu kommt, daß viele Bewohner von diversen informellen Kleinhandels- und Dienstleistungstätigkeiten leben, zu deren Ausübung sie auf einen großen Kundenkreis aus der Nachbarschaft oder die Nähe von Straßen und Gassen angewiesen sind. Viele der Siedler haben vor oder in ihren Wohnhütten nämlich gleichzeitig Krämerläden, Coiffeursalons, Wäschereien, Nähereien, Reparaturwerkstätten oder Garküchen eingerichtet. Im vierten Stock eines Mietshauses wären diese Aktivitäten wohl eines wesentlichen Anteils ihrer Kundschaft beraubt und damit kaum mehr lukrativ.

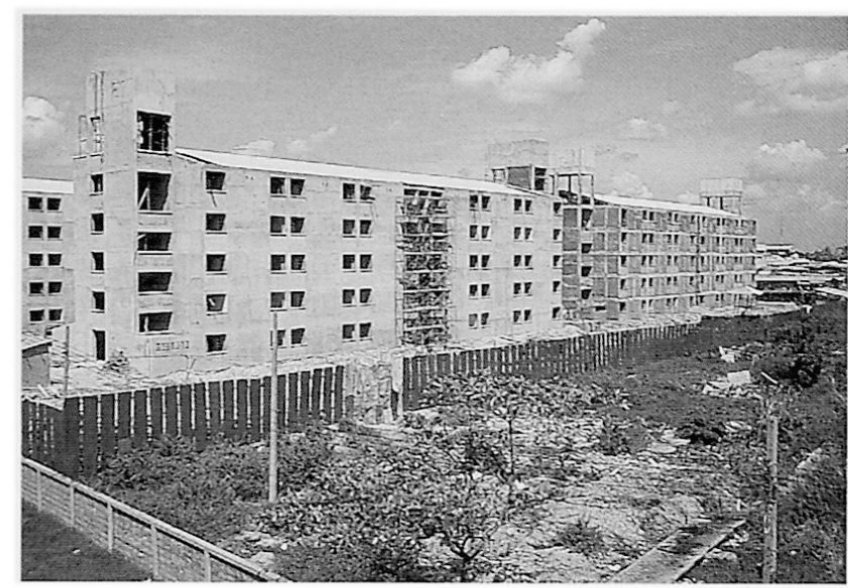

Abb. 2 Wohnblöcke: meist unbeliebte Alternative zum Leben in Holzbehausungen.

Bei der Errichtung der Siedlung «70 Rai» scheinen die Planer die Bedürfnisse der Bewohner besser berücksichtigt zu haben. Doch auch hier gibt die Bauweise Anlaß zu Kritik seitens der Bewohner: Das betonierte Fundament der Häuser befindet sich in direktem Kontakt mit dem Boden, was die Luftzirkulation unterbindet und die Parterreetagen während der Regenzeit chronisch überfluten läßt. Viele der Häuser sind außerdem von Mauern umgeben, die zwar einerseits Schutz bieten, aber die beliebten Haus-zu-Haus-Gespräche erschweren. Über zuwenig Platz beklagen sich die Familien hier allerdings kaum, und nicht selten können gar noch ein, zwei Räume untervermietet werden.

Die Beispiele aus den Wohnblocks, den Hüttensiedlungen und der Siedlung «70 Rai» zeigen, daß der Slumkomplex Klong Toey alles andere als homogen bezeichnet werden kann. Die in unterschiedlichem politischem Kontext entstandenen Quartiere bieten heute verschiedene Rahmenbedingungen, die aufgrund ihres ermöglichenden oder verhindernden Charakters die Tätigkeiten ihrer Einwohner strukturieren. Dies gilt sowohl für sogenannte produktive wie auch nichtproduktive Tätigkeiten. $\mathrm{Da}$ diese eng miteinander verflochten und oft gar nicht voneinander zu trennen sind, soll nun anhand informeller Hafenarbeiter exemplarisch gezeigt werden.

\section{Verflechtungen zwischen Arbeit und Privatleben: das Beispiel informeller Hafenarbeiter}

Durchschnittlich finden pro Tag schätzungsweise 3000 Personen auf informellem Weg eine Beschäftigung als Lastträger im Hafen Klong Toey, rund 5000 bis 6000 sind 
Abb.3 Ausschnitte aus dem Arbeits- und Privatleben von Slumbewohnern (Aufnahmen Marco Pronk):

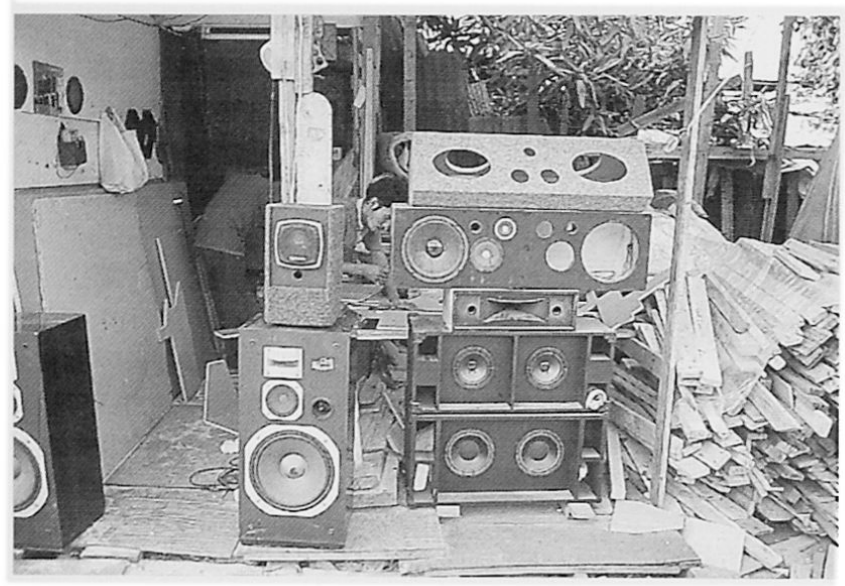

Abb. 3a Hightechproduktion im Slum: Handfertigung von Lautsprecherboxen.

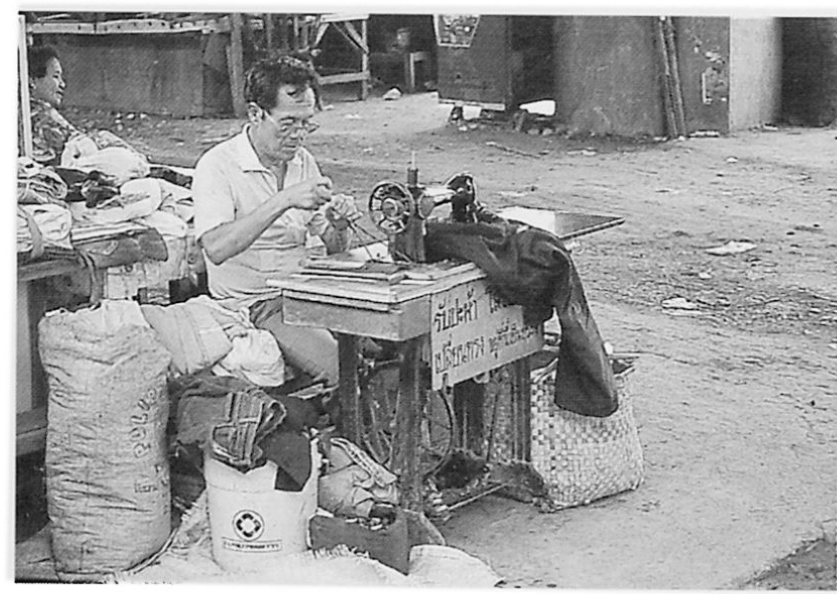

Abb. 3c Näherei am Straßenrand - gute Erreichbarkeit ist von großer Bedeutung für die Rentabilität vieler einkommensrelevanter Tätigkeiten.

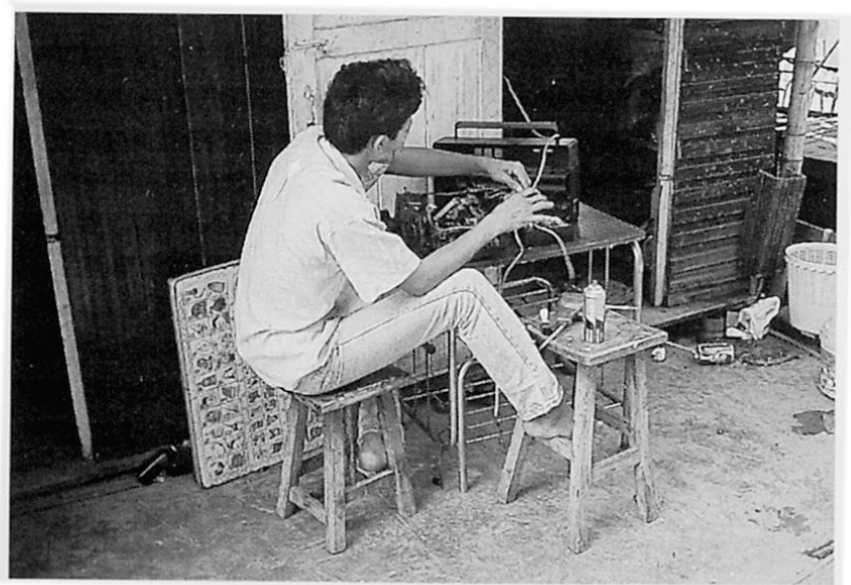

Abb.3e Reparatur von Transistorradios in hauseigener Werkstätte.

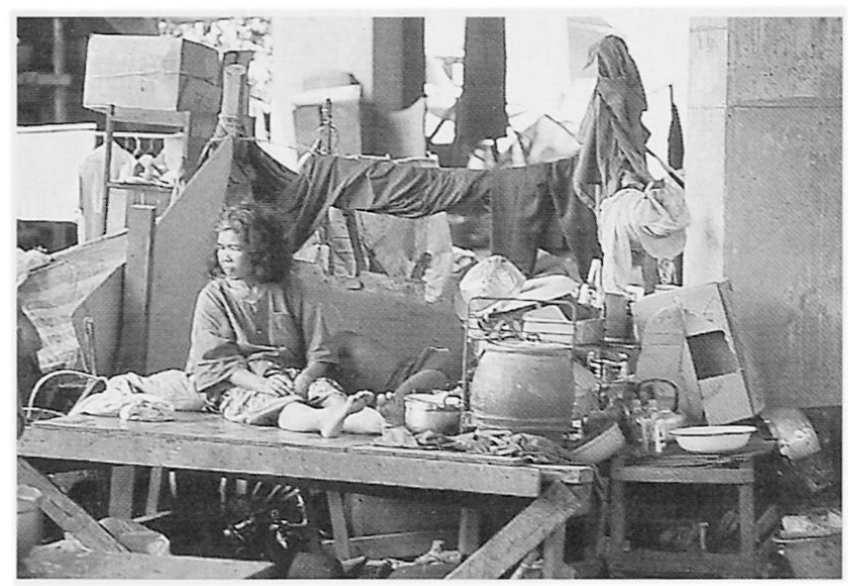

Abb. 3b Geringe Privatsphäre versus intensive Nachbarschaftsbeziehungen - für viele Slumbewohner bedeuten Bretter und Tücher inr Zuhause.

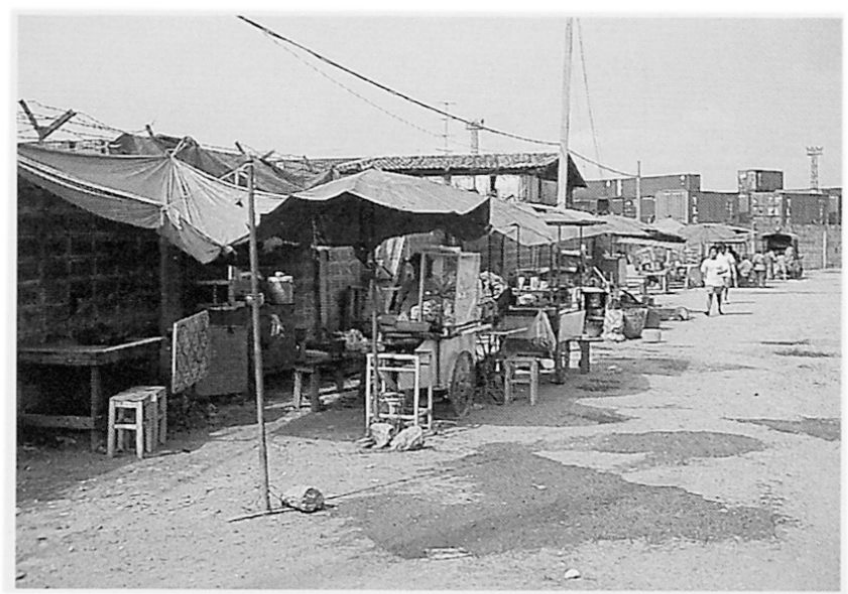

Abb.3d Garküchen vor dem Hafengelände versorgen Slumbewohner, Händler und Funktionäre des Hafenbetriebs.

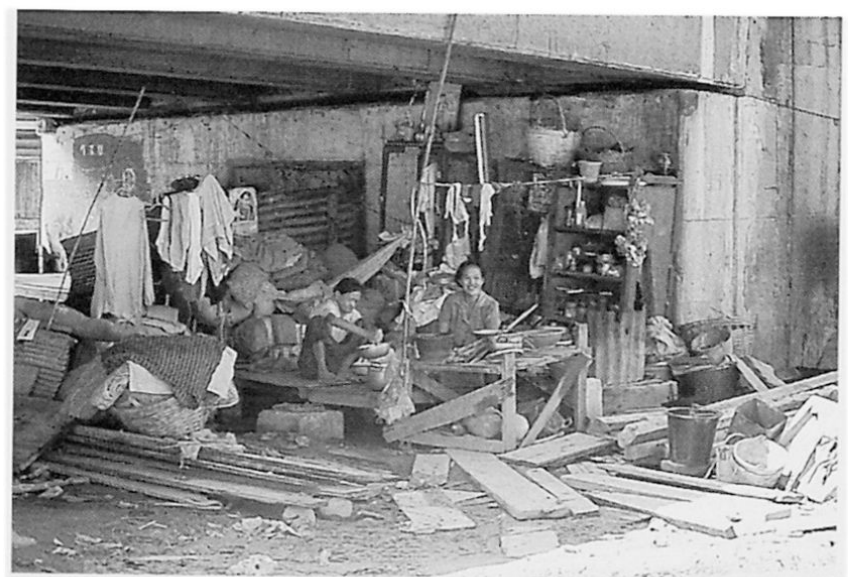

Abb. $3 f$ Bewohnerinnen unter der Stadtautobahn: Eine Brücke wird als Dach genutzt. 
tatsächlich auf Arbeitsuche. ${ }^{9}$ Nur ungefähr die Hälfte der informellen Arbeitsuchenden erhält also gewöhnlicherweise auch einen Auftrag und damit ein Einkommen. Informelle Lastträger werden im Prozeß der Güterabfertigung an den verschiedensten Stellen eingesetzt, überall dort, wo Muskelkraft noch nicht durch Maschinen ersetzt wird oder werden kann: beim Löschen der Schiffsfracht, beim Umlad von oder auf Lastwagen, in Lagerhallen bei der Zwischenlagerung von Gütern oder bei der Zollkontrolle. Nicht selten begleiten die Kulis Importwaren an ihren Bestimmungsort oder holen Exportgüter an der Produktionsstätte ab.

\section{Arbeitvergabe und Arbeitsuche}

Für Tragarbeiter im Hafen Klong Toey gibt es weder eine zentrale Meldestelle noch eine Vermittlungsstelle. Die Arbeitnehmer müssen sich selbst so organisieren, um für potentielle Arbeitgeber verfügbar zu sein. Grundsätzlich gilt, daß das Arbeitsverhältnis für die Erledigung eines Auftrags festgelegt wird, nie aber für eine fixierte Zeitdauer. Aufträge können eine halbe Stunde oder aber auch mehrere Tage dauern. Arbeitgeber der informellen Arbeiter im Hafen Klong Toey sind entweder die PAT selbst oder staatliche bzw. private Transport- und Reedereigesellschaften. Viele der für den Transport der Waren und damit auch für das Anheuern von Kulis verantwortlichen Vorarbeiter von Transportfirmen und Reedereien wohnen ebenfalls im Slum Klong Toey und kennen die Lastträger als Nachbarn, ehemalige Schulkameraden oder Freunde persönlich. Dies hat zur Folge, daß bei der Arbeitvergabe persönliche Präferenzen, Freundschaftsdienste oder Schuldbegleichungen eher zum Auswahlkriterium werden als abstrakt formulierte Anforderungsprofile.

In der Regel steht den Vorarbeitern, auch Agenten genannt, ein von der Natur und Größe der Fracht abhängiger Fixbetrag als Entgelt für die Tragarbeiten zur Verfügung, über dessen Verwendung sie gegenüber ihrer Firma meist keine Rechenschaft abzulegen haben. Da die Entlöhnung der Agenten eher bescheiden ist, ist die Versuchung oft groß, den Lohnaufwand für Kulis niedrig zu halten und die Differenz selbst zu kassieren. Dies kann auf zwei verschiedenen Wegen erreicht werden.

Erstens können die Agenten eine möglichst kleine Zahl von Arbeitern einstellen. Dieser Taktik wirkt der enorme Zeitdruck beim Verlad entgegen: Schiffe dürfen - unabhängig von ihrer Ladung und Größe - maximal 36 Stunden im Hafen Klong Toey anlegen und müssen hernach die Anlegestelle sofort verlassen, selbst wenn die Fracht noch nicht vollständig umgeschlagen werden konnte. Der Agent riskiert in solchen Fällen den Verlust seiner festen Anstellung und des Prestiges, das damit verbunden ist. ${ }^{10}$

Eine zweite Möglichkeit, einen Anteil des Fixbetrags abzuzweigen, bietet sich durch Drücken der Löhne. Die Höhe des auszuzahlenden Lohnes liegt im Ermessen des Vorarbeiters und wird mit den Kulis zum voraus ausge- handelt. Da die Anzahl der Arbeitsuchenden wesentlich höher ist als die zur Verfügung stehenden Arbeitsplätze, können die Löhne tatsächlich weit hinuntergehandelt werden. Sicherheitsüberlegungen in Verbindung mit sozialen Beziehungen sind jedoch nicht zu unterschätzende Faktoren im Prozeß der Arbeitsverteilung und -preisbildung. Der Agent ist darauf bedacht, die Fracht sicher und ohne Verlust abzufertigen. Bei wertvoller Fracht ist das Diebstahlrisiko hoch, zerbrechliche Fracht kann bei unsachgemäßer Behandlung durch unerfahrene Arbeiter zu hohen Verlusten führen. Deshalb zieht der Arbeitgeber es in vielen Fällen vor, solche Güter bekannten und zuverlässigen Lastträgern anzuvertrauen. Diese sind entweder allgemein als korrekte und vorsichtige Arbeiter bekannt, wofür er sie entsprechend besser entlöhnen muß, oder aber es handelt sich um Bekannte aus dem Slum, welche es sich nicht leisten könnten, ihn zu bestehlen, da sie damit ihren Ruf in ihrer Wohnumgebung aufs Spiel setzen würden.

Auch andere Faktoren mögen in den Entscheidungen bei der Arbeitsvergabe mitspielen: Es ist denkbar, daß ein Agent über die finanzielle Situation des Lastträgers orientiert ist und dies bei der Arbeitvergabe berücksichtigt, sei es aus Freundschaft oder aufgrund des sozialen Drucks im Slum. Andererseits mag es vorkommen, daß ein Arbeitgeber am Vorabend von einem Kuli gekränkt wurde und er diesen deshalb nun meidet.

\section{Die Bedeutung der Gruppen}

Der Intransparenz und Unberechenbarkeit des Arbeitsmarktes begegnen viele der informellen Lastträger mit eigenen Organisationsformen: Nur wenige streifen als Einzelgänger durch das Hafenareal. Die meisten Arbeitsuchenden formieren sich in Gruppen von durchschnittlich fünf bis zehn Kulis. Als Gruppe finden sie sich jeweils an einem mehr oder weniger fixen Ort auf dem Hafenareal oder vor einem der beiden Hauptportale ein. Damit erreichen sie, daß sie für die meist über den Aufenthaltsort orientierten Agenten innert kurzer Frist auffindbar und abrufbar sind. Auch in diesem Zusammenhang wird deutlich, daß bestehende Kontakte aus dem Slum von zentraler Bedeutung sind: Je zahlreicher und besser die Beziehungen einzelner Gruppenmitglieder zu Agenten oder anderen Kulis, desto größer sind die Chancen, von solchen aufgesucht zu werden und zu Arbeit zu gelangen. Der durch den Gruppenverband gewährte Informationsvorsprung erhöht nicht nur die Wahrscheinlichkeit von häufigeren Arbeitseinsätzen und damit auch von höheren Einkommen, sondern wirkt sich auch in höheren Abfindungen für Einzelaufträge aus: Ein Einzelgänger erhält bedeutend weniger Arbeitsaufträge und ist deshalb gezwungen, jede Gelegenheit zu ergreifen. Zudem kann er im Falle von Einkommensausfällen nicht auf die Solidarität anderer zählen. Seine schwache Verhandlungsbasis kann er meist nur mit der Bereitschaft kompensieren, für einen unterdurchschnittlichen Lohn zu arbeiten. So 


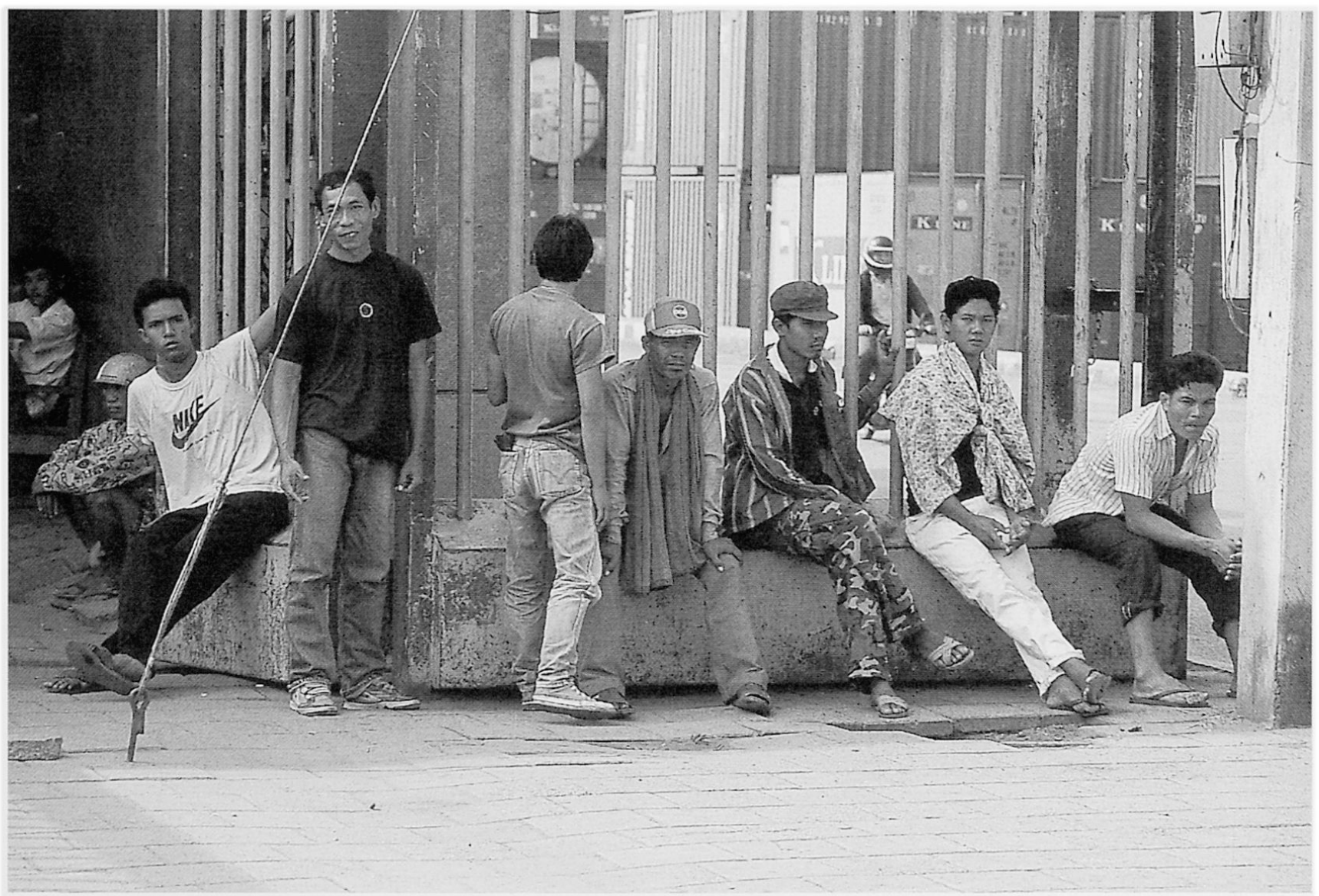

Abb. 4 Eine Gruppe von Lastträgern wartet auf Arbeitsaufträge vor dem Ostportal.

erklären sich viele auf sich allein Gestellte bereit, einen Tagesauftrag gegen ein Entgelt von lediglich 80 bis 100 Baht ( 4 bis 5 Fr.) anzunehmen, während die in Gruppen organisierten Kulis dieselbe Arbeit für einen Lohn nicht unter 150 Baht (ca. 7.50 Fr.) verrichten würden. Meist sind sie besser über Preise orientiert, die ein Auftraggeber allenfalls zu bezahlen bereit ist. Zudem steht dieser oft unter Druck, seinen Ruf im Privatleben aufs Spiel zu setzen, wenn er seinen Bekannten und Nachbarn Hungerlöhne bezahlt. An arbeitsreichen Tagen liegen für die Angehörigen eines Gruppenverbands individuelle Einnahmen von 400 bis 500 Baht (20 bis 25 Fr.), in Extremfällen gar bis zu 800 Baht (ca. 40 Fr.), durchaus im Bereich des Möglichen. " Eine Hochrechnung der Tageseinkünfte auf ein durchschnittliches Monatseinkommen ergäbe jedoch wenig Sinn, da nur an etwa drei Tagen pro Woche tatsächlich gearbeitet werden kann. Zudem werden die Konsumgewohnheiten direkt auf das jeweilige Tageseinkommen abgestimmt. Die Berechnung eines monatlichen Durchschnitts wäre nur dann sinnvoll, wenn die Arbeiter selbst in diesen Größenordnungen rechnen würden, was keinem der Befragten attestiert werden kann.

Für die meisten Aufträge werden mehrere Arbeitskräfte benötigt, je nach Arbeit mal zwei oder auch mal zehn
Leute. Werden seitens des Auftraggebers ausdrücklich bestimmte Personen angefordert, hält sich die Gruppe an dessen Präferenz. Sonst muß in der Gruppe entschieden werden. Dabei werden oft auch Faktoren berücksichtigt, die außerhalb des Arbeitsbereichs liegen. Die Gruppenangehörigen kennen sich in der Regel schon länger: Sie sind vielleicht als Nachbarskinder zusammen aufgewachsen, waren in denselben Jugendbanden aktiv, treiben schon seit Jahren gemeinsam Sport oder kennen sich von der Schule her. Sie sind eher als eine Gruppe von Freunden unterwegs, als daß sie eine Arbeitsgemeinschaft bilden. Der Zusammenschluß zur Gruppe erfolgte meist schon vor dem Entschluß, im Hafen auf Arbeitsuche zu gehen. Im Idealfall orientiert sich die Verteilung von Arbeitsaufträgen in diesen Gruppen am kollektiven Gedächtnis: Jedes Gruppenmitglied weiß von den anderen, wie oft, wie lange und für wieviel sie in den vergangenen Tagen gearbeitet haben. Bei Arbeitsangeboten werden vorwiegend jene berücksichtigt, deren letzter Auftrag schon am längsten zurückliegt bzw. deren Einkünfte in letzter Zeit am niedrigsten gewesen sind. Sind diese Verhältnisse ausgeglichen, werden oft private Gründe geltend gemacht, indem familiäre Sonder- oder Notsituationen zum Thema gemacht werden: Gründe wie außerordentliche Ausgaben für Medizin, dringende Reparatu- 
ren am Haus, Verlust eines lebensnotwendigen Gegenstands infolge Diebstahls oder außerordentliche Anschaffungen wie Schulbücher oder -uniformen für die Kinder werden in die Diskussion eingebracht. Dabei fällt auf, daß jeweils nicht der Betroffene selbst solche Punkte zur Sprache bringt, sondern gut orientierte Gruppenmitglieder darauf aufmerksam machen. In der Regel finden die Einwände bei der Arbeitsverteilung gebührend Berücksichtigung. Die Gruppe ersetzt damit einen Teil der seitens der Arbeitgeber und des Staats fehlenden Sozialleistungen und übernimmt die Verantwortung für ein gewisses $\mathrm{Ma} ß$ an sozialer Gerechtigkeit.

Die Zusammenarbeit und der Zusammenhalt der Gruppe verlaufen allerdings nicht immer so harmonisch: Sich nicht an die Konventionen haltende Gruppenmitglieder werden beschimpft, ausgeschlossen, wenn nicht gar verprügelt. Allerdings können es sich die wenigsten wirklich leisten, die meist stillschweigend festgelegten Abmachungen zu brechen, da sie sich so nicht nur in der bedeutend nachteiligeren Situation von Einzelgängern auf dem Arbeitsmarkt wiederfinden, sondern auch im privaten Bereich Freundschaften aufs Spiel setzen.

Zudem gibt es Gruppen, in denen dominante Anführer über die Einsätze ihrer Anhänger bestimmen. Solche Führer verfügen meist über einen bedeutend größeren Bekanntenkreis und damit bessere Kontakte als ihre Mitläufer. Letztere sind meist Neuankömmlinge in Klong Toey, die sich meist nur so lange um ihren Führer scharen, bis sie selbst über genügend Kontakte verfügen oder in solidarischeren Gruppen aufgenommen werden.

Auch wenn die Gruppenbildung zahlreiche Vorteile mit sich bringt, ist es nicht allen Lastträgern vergönnt, sich einem solchen Team anzuschließen. Dies kann damit begründet werden, daß sie aufgrund kürzerer Aufenthaltsdauer in Klong Toey oder aus mangelnder Solidarität in ihrem sozialen Umfeld kaum über Kontakte verfügen. Denn das Privileg, einer Gruppe anzugehören, hängt vordergründig von der Intensität der Integration der einzelnen Personen in die Slumgemeinschaft ab und ist mit der Erfüllung verschiedener Bedingungen verbunden. Denn die wenigsten der Gruppen sind als bloße Zweckgemeinschaften zur gemeinsamen Arbeitsuche zu verstehen. Der Gruppenverband reicht meist nicht nur über die Arbeitstätigkeit hinaus, sondern hat vielmehr im Privatleben seinen Ursprung und wird dort wiederum zementiert.

\section{Verflechtungen mit dem Privatleben}

Nicht nur bei der Arbeit hat sich ein Gruppenmitglied an die Abmachungen der Gruppe zu halten, sondern auch im Privatleben. Das hohe Maß an Verflechtung zwischen beruflicher und privater Welt läßt keine getrennte Betrachtung dieser beiden Bereiche zu. Wie tagsüber, auf der Arbeitsuche im Hafenareal, halten sich die Hafenarbeiter auch in ihrer Freizeit meist an den mehr oder wenigen gleichen Orten im Slum auf. In der Regel sitzen sie abends zu einer Mahlzeit oder einer Trinkrunde beisammen. Als Treffpunkt dient entweder das Haus eines Gruppenmitglieds oder eine Stammkneipe. Die Gruppe kann so jederzeit kontaktiert werden. Denn sollte mit der Arbeit zu früher Morgenstunde begonnen werden, wenn sich normalerweise noch keine Kulis eingefunden haben, stellen die Agenten bereits am Vorabend ihre Equipen zusammen. Auch hier werden oft gezielt jene Kulis aufgesucht, die für die Arbeit geeignet scheinen. Die Erreichbarkeit der Arbeiter ist somit auch abends von großer Bedeutung. In diesem Zusammenhang spielt wiederum das Wohnumfeld eine ausschlaggebende Rolle: Wer an einer zentralen, leicht begehbaren Gasse wohnt, hat Vorteile gegenüber jenen, die in abgelegeneren Quartieren zu Hause sind, geschweige denn in Mietwohnungen untergebracht sind. Abendliches Beisammensein kann allerdings nicht als strategisches Handeln der Hafenarbeiter betrachtet werden: Sie sitzen keinesfalls bloß zusammen, um auf Aufträge zu warten. Es ist ganz einfach ein Abend unter Freunden. Das Zusammenspiel zwischen abendlicher Geselligkeit und Arbeitsuche hat sich erst im Laufe der Zeit so ergeben.

Erwähnenswert ist schließlich noch der Aspekt der Umverteilung. Damit wird die Funktion der Gruppe als Puffer für Einkommensausfälle einzelner Gruppenmitglieder angesprochen, die in gut eingespielten Gruppen stillschweigend zum Tragen kommt. Alle Gruppenmitglieder sind über die individuellen Arbeitstätigkeiten und Einnahmen informiert. Da meist nur ein Teil der Gruppe gleichentags einen Lohn empfangen kann, während der Rest leer ausgeht, sind es meist erstere, die für Mahlzeiten und Getränke bezahlen. Auch hier werden die Besonderheiten spezifischer privater Situationen berücksichtigt: Wurde bei der Arbeitsverteilung für jemand eine Notsituation geltend gemacht, muß er sich auch an der Kostenverteilung meist nicht gleichermaßen beteiligen wie jemand, dem turnusgemäß eine Arbeit zustand.

Auch hier können Uneinigkeiten auftreten und Sanktionen ergriffen werden. In jedem Fall unterliegt die Verwendung des verdienten Geldes der Kontrolle der Gruppe. Damit kann einerseits ein gewisses Maß an sozialer Gerechtigkeit innerhalb der Gruppe erreicht werden, andererseits wird den einzelnen Gruppenmitgliedern die Befriedigung individueller Bedürfnisse erschwert. Zudem werden die Familienangehörigen dadurch oft finanziell vernachlässigt: Es bleibt oft nur noch ein kleiner Bruchteil für den Haushalt übrig. Der Druck der Gruppe, oft einhergehend mit fehlendem Verantwortungsbewußtsein der Familienväter, ist wesentlich dafür mitverantwortlich, daß die Ehefrauen und deren Kinder vermehrt nach eigenen Einkommensquellen suchen. Aus dieser Tatsache muß gefolgert werden, daß die in der Sozialforschung häufig verwendete Untersuchungskategorie «Haushalt» nicht für alle Studien gleich geeignet ist. Denn die wenigsten Haushalte in Klong Toey führen eine gemeinsame Haushaltskasse. Oft wissen die einzelnen Familienmitglieder nicht darüber Bescheid, wieviel ihre Angehörigen verdienen oder besitzen. Gegenseitige 
Angaben bleiben vage oder werden untertrieben, meist aus Angst, es werde Anspruch auf einen Anteil dieses Geldes erhoben. Die Einkommenskombination aus verschiedenen Produktionstätigkeiten kann in diesem Kontext wohl kaum als «Haushaltstrategie» gewertet werden. Die einzelnen Haushaltsmitglieder orientieren sich bezüglich Geldeinnahmen und -ausgaben, zumindest unter Hafenarbeiterfamilien im Slum Klong Toey, eher an auBenstehenden Gruppen als an der Familie.

Auch auf individueller Ebene muß der Begriff «Überlebensstrategie» mit Vorsicht verwendet werden. In diesem Zusammenhang stellt sich vorerst die Frage, wo die Grenze zwischen überlebensrelevanten Grundbedürfnissen und nachrangigen Bedürfnissen liegt. Üblicherweise werden zu ersteren die zum physischen Überleben notwendigen Nahrungsmittel und angemessene hygienische Bedingungen gezählt. Unter diesem Blickwinkel würde Alkohol zum Beispiel kaum unter den dringendsten Bedürfnissen figurieren. Dabei wird allerdings übersehen, daß Alkoholkonsum allenfalls als wichtiger sozial integrierender Faktor ebenso zur Sicherung des Lebensunterhalts beitragen kann. Es soll hier auf keinen Fall dem Alkoholkonsum das Wort geredet werden. Und natürlich ist es nicht der Alkohol selbst, der den Arbeitern ihr Einkommen sichern hilft, sondern die um den Alkoholkonsum aufgebauten Gewohnheiten und Regelungen. Auf indirektem Weg kann in Klong Toey Alkoholkonsum jedenfalls Kontakte ermöglichen, die ihrerseits die Chancen auf Arbeit verbessern. Negativ formuliert bedeutet dies, daß dem Alkoholgenuß entsagende Hafenarbeiter nicht als Beisitzer an den abendlichen Trinkgelagen akzeptiert werden, was einem Rausschmiß aus der Gruppe gleichkommt. Wie gezeigt wurde, ist jedoch die Mitgliedschaft in einer Gruppe für den Einkommenserwerb von größter Bedeutung.

Nun haben die abendlichen Trinkrunden jedoch nicht bloß einen strategischen Charakter, sondern dienen in erster Linie dem Vergnügen und der Ablenkung. Daß sich diese noch mit Arbeitsuche und Umverteilungen verbinden lassen, wird aus der Sicht der Lastträger wohl eher als willkommener Nebeneffekt taxiert, als daß diese im Zentrum stehen. Nicht selten werden abends die gesamten Tageseinnahmen verpraßt. Bleibt noch Geld übrig, nehmen sich die Arbeiter auch ab und zu die Freiheit, anderntags auf Arbeitsuche zu verzichten und dafür Vergnügungen nachzugehen. Von Überleben im Sinne von Existenzkampf kann hier demnach ebensowenig die Rede sein wie von Strategie im Sinne einer berechnenden Taktik. Die Organisation in Gruppen, kollektive Entscheidungsfindung bei der Arbeitsverteilung und Umverteilungsaktionen in abendlichen Runden tragen zwar zweifellos wesentlich zur Existenzsicherung bei. Sie sind jedoch eher als eine aufgrund spezieller Rahmenbedingungen im Umfeld von Slum und Hafen allmählich entwickelte, routinisierte Lebensweise zu verstehen.

\section{Zusammenfassung und Schlußfolgerungen}

Die Heterogenität des Slums Klong Toey bezüglich der Aktivitäten und der Wohnsituation und die Verflechtungen mit Welthandel, nationaler und städtischer Politik lassen keine ganzheitliche Analyse des Slums als geschlossene Gemeinschaft zu. Die aufgrund ihrer unterschiedlichen Entwicklungsgeschichte differierenden Rahmenbedingungen in den einzelnen Slumquartieren strukturieren die Tätigkeiten und Beziehungen der Siedler in verschiedenen Formen. Die Erfolglosigkeit von Zwangsumsiedlungen ist in erster Linie dadurch zu begründen, daß die Betroffenen sich in neuen Strukturen zurechtfinden müssen, welche eine herkömmliche Lebensweise nicht mehr erlauben. Für das Zusammenleben zentrale Beziehungsgefüge werden zerstört und für die Existenzsicherung wichtige Produktionsformen verunmöglicht.

Wie das Beispiel informeller Lastträger im Hafen Klong Toey zeigt, sind Verflechtungen zwischen privaten und arbeitsbezogenen Bereichen des Alltags von großer Bedeutung. Wer privat unsolidarisch und entgegen üblichen Konventionen handelt, hat wesentlich kleinere Aussichten auf regelmäßige Anstellungen im Hafenbetrieb. Zudem liegen die Löhne für im Slum unbekannte, über wenig soziale Beziehungen verfügende Arbeiter bedeutend tiefer als für alteingesessene Kulis mit etablierten Kontakten. Der Zusammenschluß zu Gruppen kann dazu beitragen, das Beziehungsnetz einzelner Lastträger zu erweitern. Ferner finden die Hafenarbeiter in den Gruppen Ersatz für sonst fehlende Sozialleistungen. Die Bildung von Gruppenverbänden als Überlebensstrategie zu taxieren wäre allerdings verfehlt, wird die Gruppenbildung aus der Sicht der Arbeiter doch eher als Akt der Freundschaft als eine Taktik der Einkommenssicherung verstanden. Der Slumbewohnern oft zum vornherein attestierte, dauernde Existenzkampf muß relativiert werden, da es auch ihnen in Phasen besserer Einkommensverhältnisse durchaus möglich ist, sich zu amüsieren. Unter dem Druck der Gruppe ist dies allerdings oft nur auf Kosten individueller Bedürfnisbefriedigung oder der Vernachlässigung von Familienverpflichtungen möglich. In jedem Fall sind die Arbeits- und die Privatsphäre der Bewohner des Slums Klong Toey dermaßen eng miteinander verflochten, daß deren getrennte Analyse kaum zum Verständnis von Bedürftigkeit und Armut führen kann. In diesem Sinne ist der vorliegende Artikel als ein Plädoyer zu verstehen, in der Entwicklungsforschung vermehrt die Verknüpfungen zwischen den verschiedenen Aspekten alltäglicher Lebensweisen zu berücksichtigen. Nur so können angemessene Maßnahmen ergriffen werden, die den Lebensformen und Bedürfnissen der Benachteiligten entsprechen. 


\section{Anmerkungen}

' Der Artikel basiert auf der Diplomarbeit des Autors: "Soziale und wirtschaftliche Verflechtungen im Alltag von Slumbewohnern in Bangkok - analysiert am Beispielvon informellen Hafenarbeitern im Slum Klong Toey" (1994), ausgeführt am Geographischen Institut der Universität Zürich. Die empirischen Erhebungen wurden im Sommer/Herbst 1992 während eines dreimonatigen Aufenthalts im Slum Klong Toey mit qualitativen Methoden (teilnehmende Beobachtung, narrative und semistrukturierte Interviews) durchgeführt.

${ }^{2}$ Zum Beispiel die Autoren ELWERT, EVERS, KORFF, SCHIEL, WILKENS u. a.m.

3 "Schattenwirtschaft" gilt als der übergeordnete Begriff, der alle wirtschaftlichen Tätigkeiten umfaßt, die nicht in offiziellen Statistiken erfaßt sind und deshalb außerhalb des staatlichen Kontroll-, Schutz- und Besteuerungsbereichs liegen (EVERS 1991: 34). Die Schattenwirtschaft setzt sich aus den «informellen Tätigkeiten" und der "Subsistenzproduktion" zusammen. Die informellen Tätigkeiten ihrerseits umfassen alle wirtschaftlichen Tätigkeiten, die Güter oder Dienstleistungen für den Markt produzieren bzw. anbieten. Die Subsistenzproduktion ist jener Teil der Schattenwirtschaft, in welchem eigengebrauchsorientiert und außerhalb von Marktzusammenhängen produziert wird (EVERS 1987: 138).

${ }^{4} 70$ Rai = ca. 11,2 ha (1 Rai = ca. 0,16 ha).

${ }^{5}$ Wechselkurs September 1992: 1 SFr. $=19.82$ Baht.

${ }^{6}$ Dieser einmalige Betrag betrifft nur das Recht auf Einzug in die Wohnung und geht direkt an die Familie, der die Wohnung im Rahmen einer Umsiedlung zugesprochen wurde. Er ist unabhängig von der Monatsmiete, die 1992 einheitlich 338 Baht (ca. 17 Fr.) betrug.

${ }^{7}$ Die Monatsmiete beläuft sich in diesem Slumquartier auf 120 bis 2000 Baht (ca. 6 bis 100 Fr.), abhängig von der Nähe zur Straße.

${ }^{8}$ Im Alltagssprachgebrauch werden Nachbarn mit "phüan ban" bezeichnet, was wörtlich übersetzt soviel wie "Freunde des Hauses" bedeutet.

${ }^{9}$ Da informelle Hafenarbeiter nicht registriert werden, beruhen diese Angaben auf Schätzungen. Die Angaben eines Sprechers der Hafenbehörden und eines Sprechers der Slum Klong Toey Federation decken sich ungefähr.

${ }^{10}$ Viele der Agenten haben früher selbst als informelle Lastträger gearbeitet. Eine feste Anstellung bedeutet für die meisten größere ökonomische Sicherheit und hohes Ansehen im Slum.
"Solche Gelegenheiten bieten sich nach Angaben mehrerer Informanten allerdings durchschnittlich nur einmal innert drei bis vier Wochen.

\section{Literatur}

BOURDIEU, P. (1991): Physischer, sozialer und angeeigneter Raum. In: Stadt-Räume (Hrsg. M. Wentz), Campus-Verlag, Frankfurt am Main.

ELWERT, G./EVERS, H.-D./ WILKENS, W. (1983): Die Suche nach Sicherheit: Kombinierte Produktionsformen im sogenannten informellen Sektor. In: Zeitschrift für Soziologie, Nr. 4, 281-296.

EVERS, H.-D. (1987): Subsistenzproduktion, Markt und Staat Der sogenannte Bielefelder Verflechtungsansatz. In: Geographische Rundschau, Nr. 39, 136-140.

EVERS, H.-D. (1991): Shadow Economy, Subsistence Production and Informal Sector: Economic Activity Outside of Market and State. In: Prisma, Nr. 51, 34-45.

IGEL, B. (1988): Die Überlebensökonomie der Slumbewohner in Thailand. Territorium und Charakteristika einer "eingebetteten" Wirtschaft. Inaugural-Dissertation, Fachbereich Wirtschaftswissenschaft, Freie Universität Berlin.

KOMLAWAN KULAHBWONG (1992): Naeo nohm gahn damnoen ngahn nai thasawat 1990 gahn tha rüa (Richtlinien für die Hafenverwaltung bezüglich der Entwicklung in den neunziger Jahren). In: Tha Rüa, 39. Jahrgang, Nr. 393, Mai-Juni 1992 (in Thai).

KORFF, R. (1991): Die Weltstadt zwischen globaler Gesellschaft und Lokalitäten. In: Zeitschrift für Soziologie, Jg. 20 Heft 5, S. 357-368, Sonderdruck, F. Enke Verlag, Stuttgart, Oktober 1991.

KORFF, R. (1992): Markets, Trade and State:Urbanism in Thailand. Fakultät für Soziologie, Universität Bielefeld, Bielefeld.

MENZEL, U. (1992): Das Ende der Dritten Welt und das Scheitern der großen Theorie. Suhrkamp Verlag, Frankfurt am Main.

PORT AUTHORITY OF THAILAND I (O. Jg.): The Port Authority of Thailand. Introduction. Publicity and Library Section, Technical Office, Bangkok.

SAMAPHAN CHUMCHON-AE-AD KLONG TOEY (1992): Sarup gahn samana prajam-pee 2535, Krungthep (Slum Klong Toey Federation [1992]: Abstracts of the Annual Seminar 1992, Bangkok) (in Thai).

WERLEN, B. (1993): Society, Action and Space. An alternative human geography. Routledge, London/New York. 